\title{
Verbesserungspotential beim Antibiotikaeinsatz
}

\section{Das Interview führte: Matthias Scholer}

Online- und Printredaktor SÄZ

\begin{abstract}
In der Humanmedizin werden die meisten Antibiotika durch Hausärztinnen und Hausärzte verschrieben. Im Kampf gegen die Resistenzentwicklung muss deshalb auch der gängigen Verschreibungspraxis in der ärztlichen Grundversorgung Beachtung geschenkt werden. Eine in der Schweiz durchgeführte landesweite Studie zeigt, dass es in den Hausarztpraxen durchaus Verbesserungspotential beim Antibiotikaeinsatz gibt.
\end{abstract}

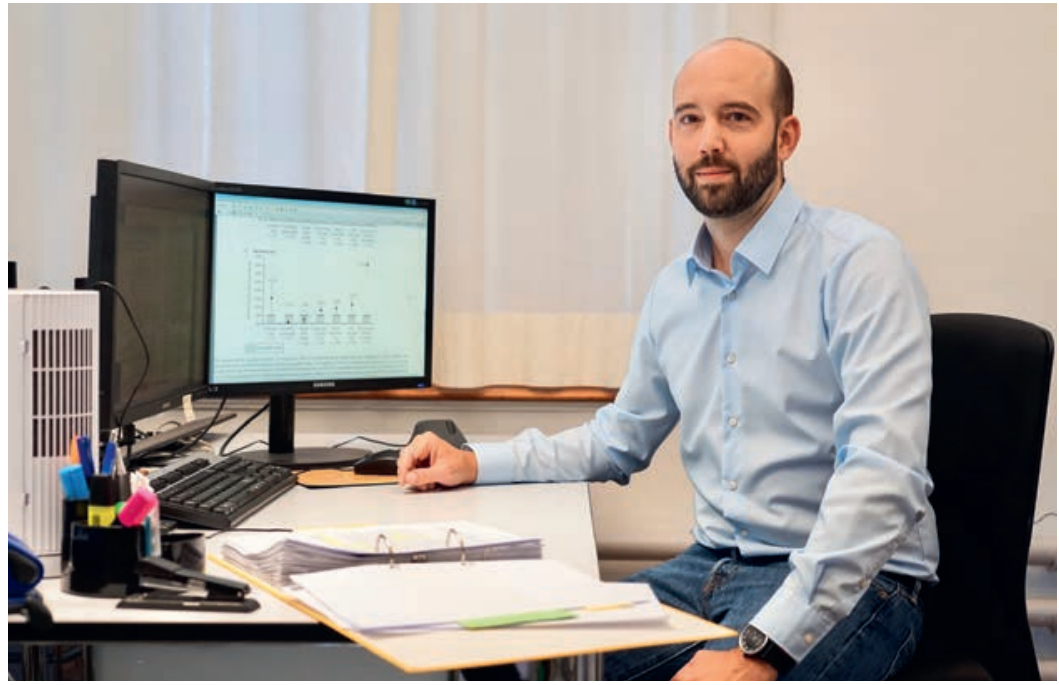

Dominik Glinz untersuchte die Antibiotika-Verschreibung in der Grundversorgung.

Herr Glinz, Sie haben in einer im letzten Jahr veröffentlichten Studie [1] die Antibiotika-Verschreibungen unserer Hausärztinnen und Hausärzte etwas genauer unter die Lupe genommen. Welche Schlüsse konnten Sie daraus ziehen?

Unsere Studie zeigte, dass die Antibiotikaverschreibungen für Infekte der oberen und unteren Atemwege und unkomplizierte Harnwegsinfekte angemessen sind, zum Teil sogar vorbildlich. Leider mussten wir aber auch feststellen, dass für einzelne Krankheitsbilder die Qualität des Antibiotikaeinsatzes zu gering ist. Dies bedeutet, dass zu oft Antibiotika verschrieben und oft- mals die falschen Antibiotika eingesetzt werden. Im internationalen Vergleich verschreiben die Schweizer Hausärztinnen und Hausärzte am wenigsten Antibiotika, es gibt dennoch vereinzelt Verbesserungspotential.

\section{Bei welchen Krankheitsbildern verzeichneten Sie einen übermässigen Antibiotikaeinsatz?} Dies war insbesondere bei Tonsillitis-Pharyngitis, akuter Otitis media, Rhinosinusitis und bei akuter Bronchitis der Fall (Anm. d. Redaktion: siehe Abb. 1). Dieses Bild deckt sich übrigens mit vergleichbaren Untersuchungen in anderen europäischen Ländern.

\section{Wie erklären Sie sich diese Verschreibungspraxis?}

Diese Frage ist nicht einfach zu beantworten, weil viele Faktoren die Verschreibungspraxis beeinflussen. So spielen zum Beispiel die Erwartungen des Patienten oder eine durch den Arzt falsch antizipierte Erwartung eine wichtige Rolle. Hinzu kommt der Zeitdruck, dem die Ärztinnen und Ärzte ausgesetzt sind und dass eine klinische Diagnose immer mit einer gewissen Ungewissheit verbunden ist. Die Liste könnte noch weiter ausgeführt werden.

\section{Sehen Sie Lösungsansätze?}

Für die oben erwähnten Krankheitsbilder ist die Unterscheidung von viralen und bakteriellen Infekten mit einer klinischen Diagnose ein zentrales Problem. Die PCR-Technologien haben sich in den letzten Jahrzehnten stark entwickelt. Ein Lösungsansatz wäre, bei 


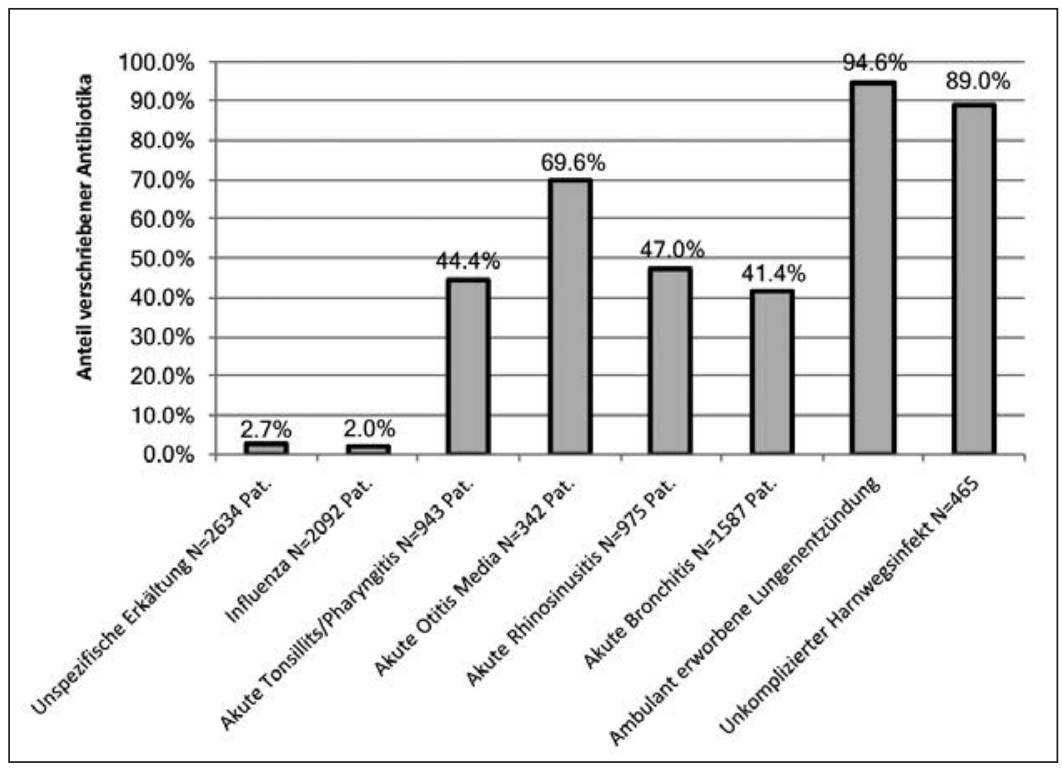

Abbildung 1: Anteil verschriebener Antibiotika aufgeteilt nach Krankheitsbildern.

diesen Krankheitsbildern eine PCR-Analyse durchzuführen um die klinische Diagnose zu unterstützen. Dies erlaubt, Virusinfektion eindeutiger von bakteriellen Infektionen abzugrenzen und entsprechend den Antibiokaeinsatz zu reduzieren. Die Fortschritte in diesem Diagnostikbereich sind beeindruckend. Heutzutage liegen PCR Resultate innert 6-12 Stunden vor. Wenn die Entwicklung so weiterläuft, ist es durchaus vorstellbar, dass in absehbarer Zeit PCR Analysen in jeder Hausarztpraxis routinemässig durchgeführt werden könnten.

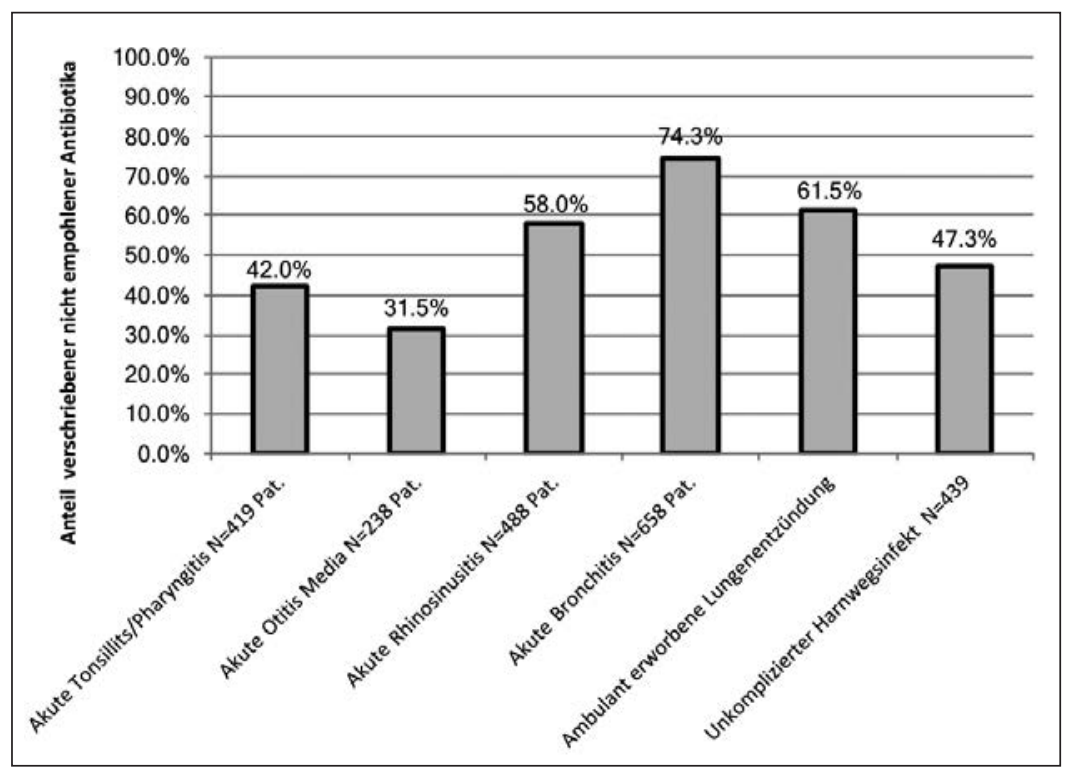

Abbildung 2: Anteil Patienten, bei denen ein nicht empfohlener antibiotischer Wirkstoff verschrieben wurde in Bezug auf das Krankheitsbild.
PCR-Analysen sind jedoch sehr viel teuer als eine Schachtel Antibiotika.

Das stimmt für die heutige Situation. Langfristig werden die PCR Analysen günstiger werden; auch sollten die Kosten, welche uns durch die Resistenzbildung entstehen, berücksichtigt werden. Doch bevor wir einen routinemässigen PCR-Einsatz diskutieren, muss zuerst untersucht werden, ob solche Diagnostikhilfsmittel überhaupt einen Einfluss auf die Verschreibungshäufigkeit haben.

In Ihrer Arbeit konnten Sie auch feststellen, dass auch die Wahl des Wirkstoffs zum Teil nicht den nationalen und internationalen Richtlinien entsprach. Inwie-

fern?

Auffallend war, dass bei fast allen untersuchten $\mathrm{Ge}$ sundheitsstörungen zu oft die falschen Antibiotika verschrieben wurden. Absoluter Spitzenreiter waren dabei Harnwegsinfekte bei Frauen, welche in 37,2 Prozent der untersuchten Fälle mit Quinolonen behandelt wurden. Im Wesentlichen stellen wir sogar fest, dass bei rund der Hälfte der Harnwegsinfekte nicht empfohlene Wirkstoffe zum Einsatz kamen. Neben den erwähnten Quinolonen sind dies insbesondere Makrolide und andere Betalaktame. Dies ist im Hinblick auf die Resistenzentwicklung bedenklich. (Anm. d. Red.: siehe $A b b$ 2.)

\section{Haben Sie eine Erklärung dafür?}

Ich persönlich bin überzeugt, dass unsere Hausärztinnen und Hausärzte grundsätzlich wissen, dass solche Back-up Antibiotika nur in Ausnahmefällen zum Einsatz kommen sollten. Über die Gründe, weshalb sie trotzdem bei auffallend vielen Fällen darauf zurückgreifen, müsste ich spekulieren. Wollen wir künftig Antworten auf solche Fragen finden, braucht es mehr Versorgungsforschung.

\section{Wie liesse sich das Verschreibungsverhalten in der Grundversorgung überhaupt ändern? Mit Repression?} Die Hausärztinnen und Hausärzte arbeiten in der Schweiz sehr autonom. Das ist gut so und soll so bleiben. Repression ist aus meiner Sicht sicherlich nicht das Mittel der Wahl.

Was wir brauchen ist ein Monitoring mit direktem Feedbacksystem. Werden zu viele oder die falschen Antibiotika eingesetzt, sollte dies in einem offenen Dialog mit den betroffenen Ärztinnen und Ärzten besprochen werden. Dies könnte beispielsweise im Rahmen von Qualitätszirkel erfolgen. 
Sie haben bei einer Studie [2] mitgearbeitet, mit der ein Monitoring-Feedback-System getestet wurde. In dieser Arbeit konnte jedoch trotz Rückmeldungen an die verschreibenden Hausärztinnen und Hausärzte keine Änderung ihrer Abgabepraxis registriert werden. Weshalb?

Bei der angesprochenen Arbeit handelt es sich um eine Machbarkeitsstudie, für die wir aggregierte Versicherungsdaten nutzten. Diese liessen nur Rückschlüsse auf die monatlichen Antibiotikaverschreibungen pro Hausärztin oder Hausarzt zu. Basierend auf diesen Daten gaben wir den Hausärztinnen und Hausärzte ein persönliches Feedback. Diese Machbarkeitsstudie war für uns die erste Erfahrung mit dem Umgang und der Nutzung von Versicherungsdaten. Die Studie war ein Erfolg, auch wenn die Feedback-Intervention keinen Effekt auf den Antibiotikaeinsatz hatte. Die aggregierten Daten haben wahrscheinlich zu wenig genau die Verschreibungspraxis der einzelnen Ärztinnen und Ärzte widergespiegelt. In einer nächsten Studie werden wir Zugang $\mathrm{zu}$ anonymisierten individuellen Patientendaten von Versicherern haben und können so den Hausärztinnen und Hausärzte ein genaueres Feedback geben.

\section{Eignen sich Versicherungsdaten überhaupt für Versorgungsforschung?}

Ja, aber wir müssen noch lernen damit umzugehen, um den grösstmöglichen Nutzen aus ihnen zu ziehen. Die Krankenkassendaten bilden zurzeit die beste Grundlage für ein flächendeckendes Monitoring. Ein möglicher Mehrwert würde sich erzielen lassen, wenn die Daten mit anderen Datenbanken verknüpft werden könnten. Im Bereich der Antibiotika könnte dies beispielsweise die Anresis-Datenbank sein, in der Resistenzdaten gesammelt werden. So liessen sich die Zusammenhänge zwischen Resistenzbildung und Verschreibungspraxis im Detail analysieren.
Wird ihrer Meinung nach in der Schweiz genug hinsichtlich Antibiotikaresistenzen unternommen?

Die Frage ist, ob wir das Richtige unternehmen. Wenn man sich vor Augen hält, dass der Antibiotikakonsum und die Resistenzentwicklung direkt korrelieren, und in der Humanmedizin bislang am meisten Antibiotika in der ärztlichen Grundversorgung verschrieben werden, müsste auch darauf fokussiert werden. Trotzdem ist in der Antibiotika-Resistenzstrategie des Bundes der Fokus auf die ärztliche Grundversorgung nur sehr vage formuliert. Es wird sich zeigen, ob in diesem Bereich die Hebel richtig angesetzt werden.

Literatur

1 Glinz D, Leon Reyes S, Saccilotto R, Widmer AF, Zeller A, Bucher HC Hemkens LG. Quality of antibiotic prescribing of Swiss primary care physicians with high prescription rates: a nationwide survey. J Antimicrob Chemother, 2017 Nov 1;72(11):3205-12.

2 Hemkens LG, Saccilotto R, Reyes SL, Glinz D, Zumbrunn T, Grolimund O, Gloy V, Raatz H, Widmer A, Zeller A, Bucher HC. Personalized Prescription Feedback Using Routinely Collected Data to Reduce Antibiotic Use in Primary Care: A Randomized Clinical Trial. JAMA Intern Med. 2017 Feb 1;177(2):176-83.

Bildnachweis

Foto: Matthias Scholer

Grafiken: Dominik Glinz

\section{World Antibiotic Awarness Week}

Auch dieses Jahr findet vom 12. bis 18. November weltweit eine "World Antibiotic Awarness Week» statt. Damit soll das Bewusstsein für Antibiotikaresistenz in der Öffentlichkeit, bei Fachpersonen und bei politischen Entscheidungsträgern gestärkt werden. In der Schweiz wird während dieser Woche unter anderem der Bund über den Stand der Umsetzung von StAR informieren und eine Kampagne starten, mit der die Bevölkerung über Antibiotika und die Resistenzproblematik sensibilisiert werden soll. 